\title{
Trayectorias sociales improbables: adolescentes chilenos de estratos bajos competentes en lectura
}

\author{
Iván Ortiz*
}

\section{Resumen}

Se ha vuelto de sentido común constatar que los logros en el aprendizaje de los estudiantes de origen social popular resultan inferiores en comparación con sus pares más privilegiados, como lo señalan las teorías de la reproducción social. Este artículo, por el contrario, fija la mirada en los casos que escapan a esta tendencia, es decir, en los estudiantes de estrato social bajo que obtienen buenos resultados académicos. Mediante análisis de regresión logística se averiguó qué tipo de factores individuales, de las escuelas y de los padres, se asocian con los estudiantes chilenos de nivel socioeconómico bajo que obtuvieron buenos resultados en la prueba de competencia lectora del estudio PISA 2009. Las variables de los estudiantes resultaron tener más fuerza predictiva que las de las escuelas y que las de los padres. Se discuten estos resultados.

\begin{abstract}
It is generally believed that students from low-income families are less successful at school, as indicated by theories of social reproduction. This article focuses on Chilean students that, in spite of their social background, have performed well in the Programme for International Student Assessment (PISA) 2009. Using logistic regression analysis we identify factors associated with academic achievement in reading. Results show that student variables have a greater explanatory value than family and school variables.
\end{abstract}

Palabras clave: educación formal, reproducción social, resiliencia, rendimiento académico, PISA.

Key words: formal education, social reproduction, resilience, academic performance, PISA.

* $\quad$ Doctor en Psicología por la Université Catholique de Louvain, Bélgica. Coordinador del área de educación técnico-profesional del Ministerio de Educación de Chile. 
ha vuelto de sentido común constatar que tanto los estudiantes de nivel socioeconómico bajo como las escuelas que los reúnen obtienen logros de aprendizaje inferiores en comparación con sus pares de niveles socioeconómicos superiores. Los resultados difundidos por los sistemas de medición del aprendizaje a gran escala, como la saga de estudios que desde hace más de una década vienen realizando el Programme for International Student Assessment (PISA) ${ }^{1}$ y el Trends in Mathematics and Science Studies (TIMSS), ${ }^{2}$ han contribuido decisivamente a ello, por cuanto han mostrado que se trata de un fenómeno casi universal, de ningún modo circunscrito a países en vías de desarrollo. Nuestro objetivo en este artículo, por el contrario, es fijar la mirada en los casos que escapan a esta tendencia, es decir, en los estudiantes de estrato social bajo que obtienen buenos resultados académicos. Estos casos revisten un gran interés para la teoría social y psicológica, así como para las políticas sociales.

Concretamente nos proponemos averiguar qué tipo de factores se asocian con los estudiantes chilenos de nivel socioeconómico (NSE) bajo que obtuvieron buenos resultados en la prueba de competencia lectora del estudio PISA 2009 (OECD, 2010a). Dicho estudio reúne gran cantidad de información adicional al rendimiento en la prueba de lectura, proveniente de cuestionarios dirigidos a los propios estudiantes, a los directores de sus escuelas y, en algunos países, a los padres de los estudiantes. Sin embargo, a pesar de que esta información adicional es interesante y pertinente, no tiene un marco conceptual integrador atingente a nuestro objeto de estudio. De modo que en estas primeras páginas, trataremos de construir una base conceptual que dé sentido a los resultados, desde un enfoque interdisciplinario que permita ampliar la unidad de análisis individual - sin perderla - ubicándola dentro de sus contextos culturales, sociales y educacionales (Cole, 2011).

Se suele citar el Informe Coleman de 1966 como el estudio emblemático que reunió evidencias cuantitativas de la determinación social del rendimiento escolar (por ejemplo, Murillo 2003). No obstante, son las teorías de la reproducción social las que han teorizado e interpretado evidencias de este tipo, integrándolas en marcos conceptuales críticos de las relaciones entre educación y sociedad. Una versión de estas teorías plantea que el sistema escolar reproduce la estratificación de la estructura social capitalista al ofrecer instituciones y opciones curriculares diferenciadas para

Programa Internacional para la Evaluación de Estudiantes.

Estudio de las Tendencias en Matemáticas y Ciencias. 
las clases sociales superiores e inferiores (Bowles y Gintis, 1981). Bourdieu y Passeron (2003) van más allá cuando argumentan que el sistema escolar enmascara la verdad de este hecho mediante la ideología meritocrática, la cual justifica el orden social que contribuye a reproducir, al exigir a todos los estudiantes un manejo del lenguaje y la cultura letrada, que en realidad se constituye fuera de la escuela, en la familia y la comunidad más cercana. Los estudiantes de los grupos sociales más cultos están en mejores condiciones de obtener éxito escolar, y luego laboral y económico, pues la escuela no hace otra cosa que transmitir la enseñanza en el código cultural de su familia. Así, estos autores ofrecen una explicación del bajo rendimiento de estos estudiantes basada en el concepto de capital cultural heredado, compuesto por el bagaje de conocimientos, léxico, disposiciones hacia la vida social y cultural que los padres transmiten a sus hijos en la socialización temprana, así como por los bienes materiales de tipo cultural (libros, cuadros, etc.) disponibles en el hogar.

Posturas como la de Bowles y Gintis, que ponen el acento en la reproducción mecánica de las desigualdades de clase a través de la escuela, no pueden explicar que no todos los hijos de padres socialmente favorecidos logran el mismo estatus, ni todos los hijos de padres de estatus social bajo siguen el mismo destino. En cambio, posturas como las de Bourdieu (1974) reparan en el hecho de que la reproducción no es total, que hay movilidades sociales que pueden observarse dentro de la estructura de desigualdades. En la medida en que describen las divergencias presentes en el lenguaje, la cultura y la práctica social de los grupos sociales, las que producen en conjunto el efecto general de reproducción, estas teorías ofrecerían una perspectiva más auspiciosa para analizar cómo operan y se constituyen tanto la permeabilidad como la impermeabilidad sociales. Pero, de hecho, ofrecen argumentos más convincentes para explicar la reproducción de la mayoría, que la excepcional movilidad de unos pocos. Bourdieu y Passeron (2003) parecen renunciar a una explicación cuando atribuyen a estos casos aptitudes particulares de los estudiantes, a su mayor adaptabilidad, o a circunstancias milagrosas.

Giroux (2004) desarrolla el concepto de resistencia, poniendo énfasis en la acción humana como elemento de resistencia al poder reproductor de la escuela. Concibe la escuela como el lugar donde las culturas dominantes y dominadas interactúan y sus ideologías conviven y se enfrentan. Dada la desigualdad de poder, las clases dominantes siempre resultan favorecidas, pero la resistencia a su dominación existe de hecho, por lo que las escuelas no son siempre funcionales al sistema imperante. Ahora bien, no todos los comportamientos de oposición pueden considerarse genuinamente como actos de resistencia; hay algunos muy ambiguos y otros que, al contrario, 
revelan una afinidad con la lógica de la dominación o la destrucción. Giroux (2004) plantea que la marca característica del comportamiento resistente es su interés subyacente emancipatorio, el cual puede tener su raíz en los valores de grupos ajenos a la escuela, como la familia o la iglesia.

Inspirados en las teorías de la reproducción social, algunos autores han prestado atención a las trayectorias y a las situaciones sociales atípicas o improbables, como el éxito escolar en las clases populares, en el marco de una preocupación mayor por la escala individual de los fenómenos sociales (Feyfant, 2011; Lahire, 1995; Mercklé, 2005). El estudio de las trayectorias atípicas sería complementario al estudio de las regularidades sociales de las cuales escapan, contribuyendo a dilucidar los mecanismos de la transmisión cultural y cómo la reproducción puede ser falseada por algunos de estos mecanismos.

Lahire (1995), por ejemplo, revela la influencia de los estilos de vida familiares, más o menos compatibles o incompatibles con el mundo escolar y sus demandas, en las trayectorias improbables. El autor encuentra hábitos domésticos de escritura en las familias populares cuyos hijos tienen éxito escolar, por ejemplo, uso de agendas, calendarios, clasificación de papeles burocráticos (cuentas, avisos), elaboración de listas para las compras, testimonios a su vez de un orden moral doméstico caracterizado por reglas estrictas de comportamiento, previsión y control del tiempo en el hogar: Todas estas disposiciones estarían ligadas también a un orden cognitivo y favorecerían la adaptación del niño(a) a la vida escolar.

Davaillon y Nauze-Fichet (2004) hicieron un seguimiento de las trayectorias escolares de niños y niñas de diferentes estratos sociales en Francia, desde el sexto grado hasta el término de la secundaria, poniendo una atención especial en la trayectoria positiva de una minoría perteneciente a los estratos bajos que, contrariamente al resto de los estudiantes de esos estratos, presentaba un rendimiento aceptable similar al promedio de los grupos más favorecidos. Encontraron en esa minoría una predominancia de padres nacidos en el extranjero, en comparación con sus pares menos exitosos; familias menos numerosas y presencia de hermanos y hermanas mayores que habían tenido una trayectoria escolar positiva. Ambos grupos no diferían en las estrategias familiares de control de los horarios cotidianos de los hijos, pero sí en la participación en la vida de la escuela: la minoría exitosa tenía algo más de vinculación y una actitud menos timorata ante los profesores. Además, estos estudiantes participaban más en las actividades extraescolares de la escuela, entre las que destacaba la mayor tasa de inscripción en la biblioteca. Todo esto a pesar de que los padres del grupo exitoso ostentaban menos niveles de escolaridad, ya que muchos de los padres y madres inmigrantes salieron de sus países de origen sin credenciales escolares. 
Con o sin inspiración en las teorías de la reproducción, el gran volumen de estudios sobre los factores asociados con el aprendizaje que aparecieron en la década de los ochenta y noventa siguió constatando una y otra vez que el rendimiento académico está fuertemente asociado con la posición social de los estudiantes. Lo más característico de esta clase de estudios ha sido buscar otros factores determinantes del aprendizaje, propios de la escuela, el hogar, la comunidad y de los mismos estudiantes, cuyo fomento pudiese compensar o revertir el impacto de las condiciones socioeconómicas de los estudiantes (Cornejo y Redondo, 2007; Murillo, 2003). Una de las corrientes más emblemáticas de esta clase de estudios es quizá la de las escuelas eficaces, que destaca el trabajo sistémico de la escuela como factor importante para el mejoramiento de los aprendizajes, aun en condiciones socialmente desfavorables. Un estudio de Bellei et al. (2004) representa un caso típico en Chile de esta corriente: destaca el aporte crucial de ciertas escuelas excepcionales en zonas de pobreza, que desarrollan expectativas altas respecto al rendimiento de los estudiantes, se enfocan en los asuntos pedagógicos y en la manutención de un clima positivo y ordenado de trabajo. Giroux (2004) proyecta la escuela como el lugar privilegiado de la resistencia, y a los profesores como agentes de emancipación, en la medida en que trabajen deliberadamente no sólo en favor del aprendizaje, sino también contra el desarrollo de necesidades alienantes en los estudiantes. Lamentablemente, es difícil encontrar en la literatura datos empíricos acerca de la extensión de una praxis educativa de esta naturaleza.

Un marco de referencia alternativo proviene del concepto de resiliencia, que alude a quienes han experimentado condiciones adversas de vida, a las cuales se han sobrepuesto gracias a una suerte de aptitud que se va forjando en la interacción de factores positivos individuales con las oportunidades que brinda el ambiente social (Manciaux et al. 2003). Bonanno (2004) dice que no se debe confundir resiliencia con recuperación, pues la primera refleja una capacidad para mantener un equilibrio estable a pesar de los eventos adversos, en vez de una recuperación rápida o total de la integridad psíquica y física después de tales eventos. De acuerdo con este autor, las personas erigen defensas frente a la adversidad, las que actúan como factores protectores. Estas protecciones pueden ser muy diversas y no siempre bien vistas por los demás, como tener un sesgo muy positivo y nada realista respecto de las propias capacidades, o bien reprimir, disociar y evitar los pensamientos, recuerdos y emociones penosos.

La investigación en resiliencia aplicada a la educación ha tendido a destacar los factores individuales asociados con el éxito escolar de los estudiantes socialmente vulnerables, aunque no ha intentado dilucidar si tales 
factores individuales pueden ser considerados causas o consecuencias de ese éxito. Finn y Rock (1997) siguieron desde el grado 8 hasta el final de la secundaria a tres grupos de estudiantes socialmente vulnerables, con buenos, regulares y malos resultados académicos; encontraron grandes diferencias entre ellos, favorables a los primeros, en cuanto al grado de compromiso e interés en las actividades escolares. Borman y Rachuba (2001) también proveen evidencia de factores individuales como el compromiso e interés con la escuela, y el locus de control interno, en estudiantes resilientes de primaria. Rouse (2001) encontró que los estudiantes resilientes creían más en su propia habilidad y en su capacidad de tener el control de su vida escolar, y menos en el apoyo del ambiente, en comparación con sus pares no resilientes. Martin y Marsch (2006) presentan evidencia de cinco factores que predicen la resiliencia académica en estudiantes de secundaria superior: confianza en las capacidades propias, control, planificación, baja ansiedad y persistencia.

El estudio de Borman y Rachuba (2001) se preocupó además de integrar variables de la escuela. Encontró que el bagaje social y académico de los pares poco aporta a las oportunidades de ser resilientes, en cambio, sí lo hacen los contextos escolares que cuentan con dispositivos que activamente protegen a los niños de la adversidad; los resilientes desarrollan vínculos de apoyo con los profesores más fuertes que sus pares resilientes, lo cual, dicen los autores, puede ser particularmente eficaz en una era en que otras instituciones comunitarias, incluyendo a la familia, son más inestables que antes.

Recientemente la Organización para la Cooperación y el Desarrollo Económico (OECD, por sus siglas en inglés) (2011) publicó un estudio internacional sobre los estudiantes de nivel socioeconómico bajo que alcanzaron rendimientos aceptables en la prueba PISA 2006 de ciencias. El estudio llama resilientes a estos estudiantes, pero no propone un marco conceptual en este sentido; los describe con base en información de los propios estudiantes y de los directores de sus escuelas, obtenida a través de sendos cuestionarios. Entre otros hallazgos, encontró que la prevalencia de los resilientes no es la misma a través de los sistemas educacionales, usando una definición comparable; en algunos países como Canadá, Australia, Finlandia, Portugal y otros, la mitad de los socialmente desfavorecidos obtienen buenos resultados en ciencias, mientras que en otros la proporción es bastante menor. Por otra parte, se identificaron dos factores particularmente bien asociados con el éxito académico de estos estudiantes: una actitud positiva hacia el aprendizaje (motivación por aprender ciencia, autoconfianza en las capacidades propias), y la cantidad de tiempo destinada a ciencias en 
el horario escolar regular, sugiriendo así que la resiliencia escolar depende de los propios estudiantes y del aprovechamiento de las oportunidades que les ofrece la escuela.

Las nociones de resiliencia y de resistencia señalan la necesidad de comprender más a fondo las formas complejas bajo las cuales la gente responde a la exclusión social, a los sucesos estresantes, a la suerte adversa. La adversidad, por dura que sea, es sólo un factor de riesgo, y el paso del riesgo al daño o al fracaso efectivos - no sólo implica una mediación que necesita ser dilucidada - sino que puede ser impedido o mitigado por factores protectores. Traspuesto a nuestro objeto de estudio, ambas nociones sugieren que los estudiantes pobres que tienen éxito escolar estarían protegidos o se defenderían de la adversidad asociada con un capital cultural heredado insuficiente o inútil, gracias a la posesión de disposiciones internas, redes de apoyo y oportunidades favorables, es decir, gracias a un verdadero capital cultural alternativo y diferente al de sus pares que fracasan. La familia jugaría un papel fundamental en la acumulación de tal capital alternativo.

Algunos podrían plantear que los estudiantes socialmente vulnerables que obtienen éxito académico son simplemente talentosos, es decir, aprendices altamente dotados. En Chile, cerca de $12 \%$ de los estudiantes que asisten a establecimientos públicos municipales, normalmente de estratos sociales bajos, son considerados talentosos por sus profesores y seleccionados para participar en programas especiales para este tipo de estudiantes (Cerda et al., 2011). Estos autores constataron una varianza importante en los puntajes de un test de inteligencia lógica entre los estudiantes de establecimientos públicos municipales, sugiriendo la presencia de altos puntajes entre ellos, pese a que en promedio los estudiantes de establecimientos privados obtuvieron puntajes más altos en el test.

Es difícil escapar a la tentación de postular un potencial innato en los estudiantes talentosos. Sin embargo, los estudiosos que investigan el rendimiento superior en diversos dominios, han desestimado la necesidad de recurrir a lo innato o a lo genético para explicar altos niveles de rendimiento, y aportan evidencia de que éste emerge después de extensos periodos de práctica deliberada, la que configura subsecuentes mecanismos cognitivos apropiados e incluso adaptaciones fisiológicas (Ericcson et al., 2007). Se ha destacado que los aprendices talentosos tienen una particular habilidad para dirigir su propio aprendizaje, con base en estrategias cognitivas (Neber y Schommer-Aikins, 2002), y que la motivación juega un papel crucial en el largo proceso a través del cual un aprendiz actualiza sus potencialidades para alcanzar excelencia en un dominio particular de actividad (Gagné, 2010). Si bien en este tipo de estudios lo que se considera talentoso es una 
performance bastante excepcional, superior al éxito académico corriente, vale la pena considerar estos antecedentes, especialmente lo relativo a la motivación y al uso de estrategias cognitivas de aprendizaje, para iluminar nuestro objeto de estudio. Cabe notar que el factor motivación aquí alude tanto a un interés genuino por aprender (Schick y Phillipson, 2009) como a tener un propósito en la vida, que orienta las metas parciales y es fuente de motivación de largo plazo (Bronk et al., 2010).

\section{Metodología}

El primer paso consistió en definir empíricamente a los estudiantes resilientes. PISA plantea dos perspectivas para definirlos, una internacional y otra nacional (OECD, 2010b). De acuerdo con la primera, un estudiante resiliente pertenece al tercio inferior de la distribución de recursos socioeconómicos de su país, pero tiene un rendimiento en el tercio superior de la distribución del rendimiento de quienes tienen una condición socioeconómica similar a la suya de todos los países. En cambio, de acuerdo con la perspectiva nacional, un estudiante es resiliente si pertenece al tercio inferior de la distribución socioeconómica de su país, y su rendimiento se ubica en el tercio superior de su país. En la perspectiva nacional, la calidad de las competencias de los resilientes puede ser muy disímil de país en país, en función de la calidad de los rendimientos globales de esos países. Como el rendimiento global de Chile fue relativamente bajo, hemos definido a los estudiantes resilientes con una perspectiva internacional, pero alternativa a la recién descrita.

Este estudio define como resilientes a quienes pertenecen a los dos quintiles inferiores de la distribución socioeconómica y alcanzan como mínimo un nivel de logro 3 en la competencia lectora. Como la distribución del ingreso en Chile es muy desigual, sigue un curso ascendente bastante plano en la primera mitad de la distribución, por lo que la vulnerabilidad social objeto de las políticas sociales suele extenderse al segundo quintil. Por otra parte, el PISA desarrolló el concepto de niveles de logro para describir las competencias que los estudiantes son capaces de poner en evidencia en las disciplinas evaluadas, dando sentido y contenido a la amplia dispersión de puntajes. Los grupos de expertos del PISA han definido el nivel 2 (de 6) como la línea de base, es decir, como el umbral a partir del cual los estudiantes empiezan a exhibir las competencias básicas para desenvolverse en el mundo de hoy y del mañana (OECD, 2010a). Por tanto, el grupo de estudiantes de NSE bajo que alcanzó el nivel 3 de logro, y algunos casos 
excepcionales, el nivel 4 (véase cuadro 1), se sitúa en un nivel de competencia lectora claramente promisorio.

Los estudiantes de los dos quintiles inferiores de NSE que sólo alcanzaron el nivel de logro 1a o 1b, constituyen el grupo de comparación de los resilientes. Llamaremos previsibles a estos estudiantes, por cuanto su bajo rendimiento es un resultado previsible de acuerdo con la literatura (denominación acuñada por Rama, 1991).

\section{Cuadro 1. Niveles de logro en lectura de los estudiantes de los dos primeros quintiles de NSE.}

\begin{tabular}{cccc}
\hline Niveles de logro & Núm. de casos en la muestra & Porcentaje & Error estándar \\
\hline Nivel 1a-1b & 944 & 43.8 & 1,97 \\
Nivel 2 & 815 & 35.6 & 1.26 \\
Nivel 3 & 406 & 17.6 & 1.14 \\
Nivel 4 & 66 & 2.8 & .42 \\
Nivel 5 & 2 & .09 & .07 \\
Total & 2233 & 100 & \\
\hline
\end{tabular}

Fuente: Elaboración propia.

La información empírica se extrajo de las bases de datos del estudio internacional PISA 2009, las cuales están disponibles en el sitio web de la institución. Las variables independientes fueron seleccionadas a partir de los cuestionarios para los estudiantes, sus padres y los directores de los colegios y corresponden a índices o escalas elaboradas a partir de varias preguntas sobre un mismo tema. Es el caso del índice de estatus económico, social y cultural, basado en diversas preguntas del cuestionario para estudiantes: escolaridad de ambos padres, ocupación de ambos padres, posesión de bienes materiales y otras. Esta y todas las escalas asociadas con las variables en estudio tienen como referente al total de la población evaluada, cuyo promedio internacional es 0 , con desviación estándar de 1 , y valores que fluctúan entre - 5 y 5 aproximadamente. Todas las variables independientes de este estudio corresponden a escalas proporcionadas por el PISA (OECD, 2010a, 2010b, 2010c) salvo la variable compañeros de NSE superior. Ésta fue construida por nosotros a partir del índice de estatus económico, social y cultural, y representa el porcentaje de compañeros de escuela pertenecientes a los quintiles 3, 4 y 5 de NSE. A continuación se presentan las variables independientes del estudio. 
- Género (masculino o femenino).

- Grado escolar: grado de la trayectoria escolar en que se encuentra el alumno a sus 15 años.

- Asistencia a educación preescolar: uno o dos años antes de entrar a primer grado.

- Sabe recordar y entender: grado en que el estudiante declara estrategias adecuadas para recordar y comprender lo que lee, que coinciden con el juicio de expertos.

- Sabe resumir: grado en que el estudiante declara estrategias adecuadas para resumir lo que lee, que coinciden con el juicio de expertos.

- Uso de estrategias de control del aprendizaje: frecuencia con que el estudiante declara usar estrategias como "me aseguro de que entiendo lo que leo", " si no entiendo algo, busco información adicional" y otras semejantes.

- Uso de estrategias de memorización: frecuencia con que el estudiante usa la memorización como estrategia de estudio.

- Uso de estrategias de elaboración: frecuencia con que el estudiante dice estudiar relacionando lo leído con otra información, o buscando aplicaciones a la vida cotidiana.

- Gusto por la lectura: grado en que el estudiante declara hábitos que implican leer por gusto y no por obligación.

- Lectura de cómics: frecuencia de lectura de cómics para disfrutar.

- Lectura de revistas: frecuencia de lectura de revistas para disfrutar.

- Lectura de ficción: frecuencia de lectura de textos literarios de ficción para disfrutar.

- Lectura no-ficcional: frecuencia de lectura de textos informativos y ensayos para disfrutar.

- Lectura de diarios: frecuencia de lectura de periódicos para disfrutar.

- Diversidad de lecturas: grado de diversidad de tipos textos y frecuencia con que el estudiante lee para disfrutar.

- Interpretación literaria en la escuela: frecuencia con que el estudiante debe interpretar textos literarios por requerimiento de la escuela.

- Textos discontinuos en la escuela: frecuencia con que el estudiante debe leer textos discontinuos por requerimiento de la escuela.

- Literatura tradicional en la escuela: frecuencia con que el estudiante sigue cursos de literatura tradicional en el establecimiento. 
- Textos funcionales en la escuela: frecuencia con que el estudiante lee textos funcionales por requerimiento de la escuela.

\section{b) Variables de la escuela:}

- $\quad$ Competencia con otras escuelas: grado en que existen en la zona establecimientos semejantes alternativos que los padres y alumnos pueden elegir.

- Selección de estudiantes: grado en que el establecimiento usa como criterio de ingreso el rendimiento académico previo de los postulantes.

- Agrupamiento por habilidad: grado en que el establecimiento agrupa a los alumnos en diferentes cursos según su rendimiento escolar.

- Índice de actividades extracurriculares: cantidad y variedad de la oferta de actividades extracurriculares que el establecimiento ofrece a los alumnos.

- $\quad$ Índice de recursos educativos: calidad y cantidad de los recursos educativos materiales que la escuela ofrece a sus estudiantes.

- $\quad$ Índice de liderazgo: grado en que el director del establecimiento dice conducir diversas tareas de gestión pedagógica del mismo.

- Pares de nse superior: porcentaje de compañeros de nse medio, medio alto y alto que tienen los estudiantes de la muestra.

- Porcentaje de niñas: proporción de la matrícula escolar de sexo femenino en el establecimiento.

c) Variables de los padres:

- Compromiso lector propio: grado en que los padres leen y están motivados con su propia lectura.

- Apoyo actual a la comprensión lectora de los hijos: grado en que los padres preguntan y discuten acerca de las lecturas actuales de sus hijos.

- Apoyo inicial a la lectura de los hijos: grado en que los padres motivaron y discutieron las lecturas de sus hijos al comienzo de su escolaridad.

- Involucramiento parental en la escuela: grado en el cual los padres participan en las actividades de las escuelas de los hijos.

- Recursos de lectura en el hogar: disponibilidad para los hijos de libros, revistas, conexión a internet y otros, distintos a los textos escolares. 
- $\quad$ Escolaridad de los padres: el grado escolar más alto alcanzado por cualquiera de los dos progenitores.

Los análisis que se realizarán exploran, primero, la distribución de estudiantes resilientes en las escuelas, para averiguar hasta qué punto ellos se concentran en determinadas escuelas, o bien comparten las mismas escuelas con sus pares previsibles. Posteriormente, se analiza en qué medida estas variables se asocian con la probabilidad de que los estudiantes de NSE bajo sean resilientes, mediante regresiones logísticas binarias (previsibles versus resilientes) aplicadas a cada grupo de variables por separado (de estudiantes, de escuelas, de padres). El análisis de regresión logística es el indicado para responder las preguntas de este estudio, por cuanto resulta útil para determinar cuáles factores están asociados con la probabilidad de un evento determinado, en este caso, que un estudiante de NSE bajo sea resiliente y no previsible. De hecho es el análisis utilizado en el estudio de estudiantes resilientes en ciencias, descrito anteriormente (OECD, 2011). Es similar a un modelo de regresión lineal pero adaptado a una variable dependiente categorial (dicotómica o multinomial). La regresión logística reporta los resultados en odds ratios (razones de probabilidad). Odds ratios mayores que uno significan que un incremento de una unidad en los valores de la variable independiente, se asocia con una mayor probabilidad de que ocurra el evento vinculado a la variable dependiente. Por ejemplo, un odd ratio igual a dos para un factor "horas de lectura" implica que un aumento de una unidad en la escala de ese factor, aumenta al doble la probabilidad de que el estudiante que posea ese puntaje sea resiliente. Si es menor que uno, la probabilidad se inclina en favor de la categoría de referencia (estudiantes previsibles, en nuestro análisis).

El PISA usa metodologías complejas que condicionan la manera en que los datos deben ser analizados (véase OECD, 2009). Para empezar, el rendimiento de los estudiantes en las pruebas de aprendizaje se calcula usando un método de imputación. En vez de calcular directamente la habilidad de los estudiantes, mediante un puntaje único, se estima una distribución de probabilidades para cada uno de ellos, es decir, un rango de habilidad que un estudiante podría razonablemente tener. De ese rango, el PISA entrega cinco valores plausibles escogidos al azar para cada estudiante, de tal modo que cualquier análisis relacionado con el rendimiento debe desarrollarse cinco veces, siendo su promedio el resultado final. En segundo lugar, el error estándar es calculado con gran precisión, mediante operaciones adicionales a las que entregan por defecto los paquetes estadísticos. Una última precaución son los pesos que deben ser asociados con cada estudiante 
y con cada escuela. En nuestro caso, los cinco valores plausibles dieron origen a cinco niveles de logro plausibles, para cada estudiante. En teoría, un estudiante puede ser asignado a diferentes niveles de logro de acuerdo con sus diferentes valores plausibles. En los hechos, pudimos efectivamente constatar que, con cierta frecuencia, los estudiantes tenían tres o cuatro valores plausibles en un nivel de logro, y el resto en un nivel de logro inmediatamente superior o inferior. Consecuentemente, todos los análisis se desarrollaron cinco veces, para cubrir los cinco niveles de logro plausibles: los resultados finales que se reportan corresponden a los valores promedio.

\section{Resultados}

Los estudiantes de los dos quintiles inferiores de NSE se distribuyen en 176 de los 200 establecimientos educacionales de la muestra nacional. Debe distinguirse entre el NSE de los estudiantes y el NSE predominante de los establecimientos. Así, estos 176 establecimientos no son todos socialmente vulnerables; hay estudiantes de los dos quintiles inferiores que estudian junto a compañeros de NSE superiores, en proporciones variables.

Los estudiantes resilientes, ¿tienden a concentrarse en algunos establecimientos o bien a distribuirse entre todos? Los resilientes están en 120 establecimientos, en tanto que los estudiantes previsibles están en 144. De acuerdo con el tamaño de cada uno de estos grupos (véase cuadro 1), la distribución más dispersa corresponde a los resilientes. No obstante, el hecho más corriente es que previsibles y resilientes (y estudiantes que alcanzan el nivel dos de logro) se encuentren juntos en los mismos establecimientos. Sólo 52 establecimientos tienen estudiantes previsibles o bien resilientes, pero no ambos a la vez. En la mayoría restante predomina la mezcla, en diversas proporciones, inclinada hacia uno u otro grupo.

La muestra total se concentra en establecimientos públicos y privados con financiamiento estatal; sólo cinco casos estudian en colegios privados pagados sin financiamiento estatal. En los establecimientos públicos los estudiantes previsibles alcanzan 51\% y los resilientes 15\% (el resto son estudiantes de nivel dos de logro). En los establecimientos privados con financiamiento estatal, los porcentajes cambian a $35 \%$ y $27 \%$, respectivamente.

\section{Modelo de los estudiantes}

Las variables incorporadas en el modelo predicen bien la probabilidad de ser un estudiante resiliente. La relación entre el número de casos observa- 
dos versus los predichos correctamente por el modelo es de $83.8 \%$. Según el test de bondad de ajuste de Hosmer y Lemeshow, las diferencias entre casos observados y casos predichos correctamente son estadísticamente significativas $(\mathrm{p}=.00)$, lo que indica un ajuste desfavorable de los datos al modelo. Para un buen ajuste, esas diferencias debieran ser tan pequeñas que el test las declarara no significativas. Sin embargo, el test es muy sensible al tamaño de la muestra: a mayor tamaño, mayor es la probabilidad de que diferencias pequeñas sean encontradas estadísticamente significativas. En nuestro análisis, el requerimiento de ponderación de los casos multiplicó la muestra al nivel de la población total de estudiantes de 15 años. En cambio, cuando este mismo análisis se realizó con la muestra sin ponderación, las diferencias entre los casos observados y los predichos correctamente resultaron no-significativas con el mismo test, revelando un buen ajuste del modelo. De acuerdo al R cuadrado de Nagelkerke (una versión corregida del $\mathrm{R}$ cuadrado, para regresión logística), el modelo explica 59\% de la varianza.

La variable más importante del modelo es el grado escolar que cursa el estudiante. Dada una misma edad cronológica, un año más de escolaridad aumenta cinco veces la probabilidad de un estudiante socialmente vulnerable de ser un resiliente (el parámetro $\operatorname{Exp}(\mathrm{B})$ expresa los odds ratio. Varias otras variables del modelo contribuyen también a la probabilidad de ser resiliente, pero con menos fuerza.

Las variables implicadas en el procesamiento cognitivo de lo que se lee por parte del lector, muestran un comportamiento interesante. El conocimiento de estrategias adecuadas para recordar y para resumir la información leída aumentan casi al doble la probabilidad de ser resiliente. El uso de estrategias presenta una jerarquía favorable a las de control, seguidas por las estrategias de memoria, que contribuyen levemente, mientras que el uso de estrategias de elaboración se relaciona con la probabilidad de ser un estudiante previsible en vez de resiliente.

El contraste entre las lecturas que se hacen por gusto y las que se hacen para el colegio es revelador. Leer por gusto revistas, diarios y textos de noficción, contribuye en distintos grados a la probabilidad de ser resiliente. No así la lectura de ficción ni la de cómics, así como tampoco la lectura de diversos tipos de texto. Por otro lado, un aumento de una unidad en la escala de disfrute de la lectura, aumenta el doble esta probabilidad. En cuanto a las lecturas vinculadas con las exigencias escolares, llaman la atención el impacto negativo de los textos funcionales y la situación ambivalente de la literatura. Respecto de esta última, interpretar los textos literarios en clases aporta a la probabilidad de ser resiliente, en cambio, los cursos tradicionales de literatura aparecen asociados con la probabilidad de ser un estudiante previsible. 


\begin{tabular}{|c|c|c|c|c|}
\hline Variables & $B$ & S.E & Sig. & $\operatorname{Exp}(B)$ \\
\hline Grado escolar & 1.61 & .026 & .000 & 5.02 \\
\hline Sexo masculino & -.22 & .027 & .000 & .81 \\
\hline Asistió a prescolar & & & .000 & \\
\hline Asistió 1 año o menos & .69 & .034 & .000 & 2.00 \\
\hline Asistió 2 años & .32 & .039 & .000 & 1.38 \\
\hline Lee revistas(1) & .40 & .036 & .000 & 1.51 \\
\hline Lee cómics(1) & \multicolumn{4}{|c|}{ Inestable y/o no significativo } \\
\hline Lee ficción(1) & \multicolumn{4}{|c|}{ Inestable y/o no significativo } \\
\hline Lee no ficción(1) & .42 & .031 & .000 & 1.55 \\
\hline Lee diarios(1) & .23 & .036 & .001 & 1.28 \\
\hline Sabe resumir & .62 & .015 & .000 & 1.86 \\
\hline Sabe recordar & .74 & .014 & .000 & 2.09 \\
\hline Usa control & .49 & .019 & .000 & 1.64 \\
\hline Lee diversidad & -.23 & .024 & .000 & .80 \\
\hline Usa elaboración & -.35 & .016 & .000 & .71 \\
\hline Usa memoria & .12 & .018 & .000 & 1.13 \\
\hline Lee por gusto & .72 & .021 & .000 & 2.07 \\
\hline Índice SE & .56 & .024 & .000 & 1.75 \\
\hline Interpretación literaria en la escuela & .50 & .017 & .000 & 1.65 \\
\hline Lee textos discontinuos en la escuela & .21 & .017 & .000 & 1.24 \\
\hline Literatura tradicional en la escuela & -.83 & .020 & .000 & .44 \\
\hline Lee textos funcionales en la escuela & -.16 & .016 & .000 & .85 \\
\hline Constant & -15.89 & .263 & .000 & .00 \\
\hline
\end{tabular}

Fuente: Elaboración propia.

Puede observarse que el nivel socioeconómico del estudiante, aun dentro de la escasa variabilidad de los dos primeros quintiles, aumenta la probabilidad de ser un estudiante resiliente, al igual que la asistencia a educación preescolar. Por otra parte, las mujeres tienen más probabilidades que los hombres de ser resilientes, aunque más bien leves.

Las variables no significativas ( $p=.051$ y más) y/o las inestables, es decir, que resultaron tener una influencia positiva en algunos de los cinco 
análisis correspondientes a los cinco valores plausibles (véase metodología), y negativo en otros, se consideran fuera del modelo.

\section{Modelo de las escuelas}

Las variables de las escuelas incorporadas en el modelo predicen razonablemente bien la probabilidad de ser un estudiante; el modelo predice correctamente a $74.5 \%$ de los casos de la muestra. El test de bondad de ajuste de Hosmer y Lemeshow es desfavorable, por efecto del tamaño de la muestra; el mismo análisis sin ponderación de la muestra, resulta en una bondad de ajuste favorable. De acuerdo al R cuadrado de Nagelkerke, el modelo explica $28 \%$ de la varianza.

\section{Cuadro 3. Variables del modelo para escuelas}

\begin{tabular}{ccccc}
\hline Variables & $B$ & S.E. & Sig. & Exp(B) \\
\hline Compite con escuelas semejantes & & & .000 & \\
Compite (1) & -.361 & .026 & .000 & 0.70 \\
Compite (2) & -.674 & .039 & .000 & 0.51 \\
Selecciona por rendimiento & & & .000 & \\
Selecciona (1) & .646 & .025 & .000 & 1.91 \\
Selecciona (2) & 1.323 & .030 & .000 & 3.77 \\
Agrupa por habilidad & & & .011 & \\
Agrupa (en algunas asignaturas) & -.177 & .026 & .009 & 0.84 \\
Agrupa (en todas las asignaturas) & & No significativa & \\
Actividades extra & & .013 & .000 & 1.32 \\
$\quad$ Liderazgo & .275 & .012 & .000 & 0.91 \\
Recursos educativos & -.099 & .011 & .000 & 1.16 \\
Conducta alumnos & .143 & .000 & 1.40 \\
Porcentaje niñas & .334 & .013 & .000 & 1.01 \\
Pares NSE superior & .013 & .001 & .000 & 1.02 \\
Constant & .019 & .001 & .000 & 0.14 \\
\hline
\end{tabular}

Fuente: Elaboración propia.

Aquellos establecimientos que seleccionan a sus alumnos por sus antecedentes académicos, algunas veces o siempre, tienen más el triple de oportunidades de contar con resilientes. La variedad de la oferta de actividades extracurriculares que ofrece la escuela, la calidad de sus recursos educativos, 
así como la buena conducta habitual de los alumnos, también aumentan la probabilidad de resiliencia de los estudiantes, aunque en menor medida. Por el contrario, aquellos establecimientos que compiten con otros semejantes en la misma zona y que los padres o alumnos pueden elegir, aportan a la probabilidad de ser un estudiante previsible, lo mismo que la agrupación de alumnos por habilidad en alguna asignatura, y la calidad del liderazgo del director. Otros dos factores, la predominancia de estudiantes de sexo femenino en los establecimientos, así como la presencia de compañeros de NSE superior en el aula, hacen una contribución casi nula.

\section{Modelo de los padres y apoderados}

El modelo predice correctamente sólo a $68 \%$ de los casos de la muestra, cifra que revela una capacidad predictiva más bien débil del modelo. El test de bondad de ajuste de Hosmer y Lemeshow se comporta igual que en los dos modelos anteriores. De acuerdo al R cuadrado de Nagelkerke, el modelo explica 14\% de la varianza.

\section{Cuadro 4. Variables del modelo para padres}

\begin{tabular}{ccccc}
\hline Variables & B & S.E. & Sig. & Exp(B) \\
\hline Escolaridad de los padres & & & 0.00 & \\
Escolaridad de los padres (ISCED 3A) & .682 & .021 & 0.00 & 1.98 \\
Escolaridad de los padres (ISCED 4) & .772 & .036 & 0.00 & 2.17 \\
Escolaridad de los padres (ISCED 5B) & .465 & .051 & 0.00 & 1.61 \\
Escolaridad de los padres (ISCED 5A-6) & -.497 & .092 & 0.03 & 0.63 \\
Sala cuna (1) & -.172 & .022 & 0.00 & 0.85 \\
Cuánto Lee & & & 0.00 & \\
Cuánto Lee (+10 horas) & -.723 & .045 & 0.00 & 0.49 \\
Cuánto Lee (6-10 horas) & .306 & .032 & 0.00 & 1.36 \\
Cuánto Lee (1-5 horas) & & Inestable y/o no significativo & \\
Motivación por la lectura propia & .070 & .011 & 0.00 & 1.07 \\
Apoyo temprano a la lectura & .235 & .011 & 0.00 & 1.27 \\
Recursos de lectura en casa & .174 & .011 & 0.00 & 1.19 \\
Participación en la escuela & -.476 & .010 & 0.00 & 0.62 \\
Apoyo actual a la lectura & .094 & .011 & 0.02 & 1.10 \\
Constant & -.510 & .026 & 0.00 & 0.61 \\
\hline
\end{tabular}

Fuente: Elaboración propia. 
En comparación con los hijos de padres que sólo tienen educación básica, los hijos cuyos padres han terminado la educación media, o tienen educación superior incompleta, o han terminado una carrera en un Centro de Formación Técnica o Instituto Profesional, tienen más oportunidades de ser estudiantes resilientes. Por ejemplo, tener educación media completa (la escolaridad más corriente en esta muestra, después de la básica) aumenta $98 \%$ esta probabilidad. Curiosamente, la escolaridad universitaria, rara en este grupo, se comporta erráticamente: predice mejor a los estudiantes previsibles.

El apoyo temprano de los padres a la lectura de los hijos, también contribuye a la probabilidad de ser resiliente: subir una unidad en esta variable, implica un aumento de $27 \%$ de esta probabilidad. La disponibilidad de recursos de lectura en el hogar también hace una contribución, pero en menor medida. La motivación de los padres por su propia lectura, contribuye casi nada, lo mismo que el apoyo actual a la lectura de los hijos. Cuanto leen en casa los padres para entretenerse, tiene una contribución contradictoria. Leer más de 10 horas a la semana (poco común en esta muestra), predice a los estudiantes previsibles; leer 6 a 10 horas a la semana, aumenta $36 \%$ las probabilidades de ser resilientes; leer poco ( 1 a 5 horas a la semana) resultó no significativo. La mayor participación actual de los padres en las escuelas de sus hijos predice, en cambio, a los estudiantes previsibles, lo mismo que el haber asistido a sala cuna o guardería en la niñez temprana.

\section{Discusión y conclusiones}

De los tres grupos de variables sometidos al análisis de regresión logística, el grupo de variables de los estudiantes a nivel individual constituyó el modelo más robusto. El grupo de variables de los padres, fue el más débil, mientras que las variables de la escuela se encuentran en una situación intermedia, pero conformando un modelo de robustez suficiente.

Las variables que resultaron tener los mayores poderes predictivos de la resiliencia, son el grado escolar del estudiante, y la selección académica por parte del establecimiento educacional. A continuación, le siguen algunas variables relacionadas con el procesamiento cognitivo de lo que leen los estudiantes, así como el gusto por la lectura. En este grupo se pueden considerar también la escolaridad de los padres, la asistencia a educación prescolar y el nivel socioeconómico de los estudiantes. Un tercer grupo de variables, de menor poder predictivo pero significativas desde el punto de vista estadístico, integra características de la escuela tales como la conducta 
positiva del conjunto de los estudiantes y la disponibilidad de actividades extracurriculares, así como la práctica de interpretación literaria en la escuela, y el apoyo temprano de los padres a la lectura de los hijos.

Un cuarto grupo de variables reviste un interés particular, pues se asocia con la probabilidad de ser un estudiante previsible en vez de resiliente. Aquí caben, el uso de estrategias lectoras de elaboración, los cursos tradicionales de literatura en la escuela, la presencia de escuelas en los alrededores que compiten por los mismos alumnos, el agrupamiento de alumnos por habilidad en los cursos, el liderazgo del director, la mayor participación de los padres en la escuela, y la asistencia de los hijos a sala cuna .

El número de años de escolaridad que los alumnos acumulan a sus 15 años resultó decisivo para la probabilidad de ser resiliente. Un año más de escolaridad implica una considerable mayor exposición a la cultura letrada de la escuela, una fuente clave de aprendizaje de la competencia lectora. Ahora bien, los grados que aportan a la resiliencia son el décimo y undécimo.

De acuerdo con la normativa chilena de edad de ingreso al primer año escolar, a los 15 años los estudiantes estarían cursando su décimo año de escolaridad. Salvo aquellos niñas y niños que, por cumplir los 6 años de edad después de junio, tienen que esperar al año siguiente para ingresar a primero básico, por lo que al llegar a sus 15 años de edad estarán cursando su noveno año de escolaridad: menos oportunidades de ser resilientes. Los alumnos de 15 años que están en el undécimo grado no habrían cumplido con las normas de edad para el ingreso al sistema escolar. Es posible que se trate de niños precoces cuyas madres insistieron en que la escuela aceptase su ingreso antes de la edad requerida; de hecho el director del establecimiento tiene la facultad de hacer excepciones a la norma y permitir el ingreso de estos casos si lo juzga pertinente. En fin, estos resultados señalan que la escolarización es más importante que las características de las escuelas en particular en la predicción de la resiliencia.

Los estudiantes resilientes se encuentran preferentemente en establecimientos escolares que reconocen seleccionar académicamente a sus estudiantes. Como se trata de un efecto independiente del efecto del grado escolar (los efectos de todas las variables en estudio son independientes), se puede suponer que los estudiantes resilientes presentan alguna credencial de rendimiento superior a la de otros estudiantes, que los hace seleccionables. En tal caso, la asociación entre resiliencia y selección académica no tiene una dirección clara. No es el establecimiento el que haría resilientes a estos estudiantes, ya lo habrían sido al ingresar a estos colegios, si admitimos que el buen rendimiento académico que hace seleccionables a los es- 
tudiantes tiene mucho que ver con su competencia lectora. Puede también que los establecimientos selectivos contribuyan a la resiliencia por la vía de ciertas características de la oferta educativa y el clima interno, que las hace elegibles por los padres más preocupados de dar una buena educación a sus hijos, como lo sugiere el comportamiento de las variables "actividades extracurriculares (diversas)" y "conducta (buena) de los estudiantes". No puede descartarse, sin embargo, un efecto positivo de la escuela selectiva sobre la resiliencia por la vía del efecto de los pares, el cual contribuiría a que otros estudiantes de esa escuela se conviertan en resilientes gracias al aporte de sus compañeros seleccionados, al mismo tiempo que éstos mantienen o acrecientan su propia competencia.

Llama la atención en los resultados encontrados que los estudiantes resilientes no están rodeados de compañeros pertenecientes a los quintiles 3, 4 y 5 de NSE en mayor o menor medida que lo están los estudiantes previsibles. La razón no radica en la escasez de estudiantes de NSE superiores. De hecho, sólo 1.8\% de la muestra no tiene ningún compañero de NSE superior al suyo; y $60 \%$ de la muestra tiene como máximo $42 \%$ de sus compañeros de NSE superior. Por cierto, el NSE superior más frecuente es el quintil 3. Algún nivel de mixtura social existe, pero no es el efecto de los pares de NSE superior el que explica a los estudiantes resilientes; en todo caso sería un efecto de los pares de buen rendimiento, concentrados por las prácticas selectivas de los establecimientos.

El procesamiento cognitivo de lo que se lee es diferente en estudiantes previsibles y resilientes. Estos últimos serían más competentes para recordar y entender información, y para resumir lo que estudian o leen. La pregunta que surge es si el conocimiento de tales estrategias metacognitivas es un resultado de una enseñanza escolar explícita, o una adquisición por ensayo y error de los estudiantes. De las estrategias que los estudiantes dicen aplicar cuando estudian o leen, sólo las de control son algo más utilizadas por los resilientes, esto es, estrategias de aseguramiento de la comprensión de lo que se lee. En cambio, las estrategias de elaboración, que suelen ser promovidas por los docentes, son más frecuentes en los estudiantes previsibles; este resultado es similar a lo que ocurre en la muestra internacional, donde esta estrategia se asocia con los puntajes bajos en la prueba (OECD, 2010c).

Pese a la mayor contundencia predictiva del modelo de variables individuales, nos parece que la predicción de los estudiantes resilientes debe mucho a la acción de las familias. La asistencia a educación preescolar y el apoyo temprano a la lectura de los hijos, son obviamente decisiones de los padres o apoderados. Es bastante general el consenso existente acerca de la importancia que reviste la educación temprana para promover equi- 
dad educativa; las políticas de ampliación de la cobertura de este tipo de educación en Chile dan testimonio de ello. Tedesco (2010) señala que para romper el círculo vicioso de padres pobres-hijos pobres, es fundamental invertir en las familias y en la primera infancia antes del inicio de la educación formal, no sólo facilitando un desarrollo cognitivo básico mediante una sana estimulación afectiva, buena alimentación y condiciones sanitarias adecuadas, sino además posibilitando una socialización primaria adecuada mediante la cual los niños adquieran los rudimentos actitudinales y comportamentales que les permitan incorporarse a la escuela.

Asistir a escuelas selectivas, por otro lado, alude menos a un factor propiamente escolar que a la voluntad de la familia de buscar un establecimiento escolar competente para el progreso escolar de los hijos. Raczynski et al. (2010) detectaron en Chile una variedad de patrones de elección de escuela para los hijos en las familias de estrato social bajo. Entre la elección pasiva, que sigue el mandato social de mandar a los hijos a la escuela - respaldado por la ley - sin importar las características del establecimiento, la elección autoexcluyente, que deja fuera deliberadamente a escuelas que plantean exigencias académicas, económicas y disciplinarias, que los hijos o los padres no podrían cumplir, y otros patrones de elección, figura la elección por distinción y movilidad social, que implica una búsqueda activa e informada de escuelas que exhiban atributos de prestigio y excelencia, aseguren calidad educativa y oportunidades futuras para los hijos. En Chile, este tipo de escuelas suelen seleccionar a los estudiantes que ingresan en ellas y ofrecer condiciones de orden, disciplina y recursos favorables al proceso educativo. Luego, detrás de los resilientes aparece una fracción de familias de estratos bajos con un comportamiento propio de las clases medias, lo que tal vez sea una explicación sencilla para una teoría de la no reproducción. No es menor el hecho de que los padres de estas familias tengan una escolaridad y un NSE ligeramente superiores a la de los padres de los estudiantes previsibles. Tal vez su demora relativa en salir del sistema educativo revela una adhesión frustrada al mismo, proyectada luego en la realización escolar de los hijos, a la cual pueden luego dedicar energías gracias a una situación laboral menos precaria.

Sería interesante averiguar en investigaciones futuras hasta qué punto estos resultados varían en función de los contextos nacionales, de las características de sus sistemas escolares y de la mayor o menor desigualdad social de sus sociedades. 


\section{Bibliografía}

Bellei, Cristián, Luz María Pérez, Gonzalo Muñoz y Dagmar Raczinski, 2004, ¿Quién dijo que no se puede? Escuelas efectivas en sectores de pobreza, Santiago de Chile, Unicef.

Bonanno, George, 2004. “Loss, Trauma and Human Resilience. Have we Underestimated the Human Capacity to Thrive After Extremely Aversive Events?", American Psychologist, vol. 59, núm. 1, pp. 20-28.

Borman, Geoffrey y Laura Rachuba, 2001, "Academic Success Among Poor and Minority Students: An Analysis of Competing Models of School Effects", Report núm. 52, Johns Hopkins University.

Bourdieu, Pierre y Jean Claude Passeron, 2003, Los herederos. Los estudiantes y la cultura, Buenos Aires, Siglo XXI Editores.

Bourdieu, Pierre, 1974, "Avenir de classe et causalité du probable", Revue Française de Sociologie, vol. 15, núm. 1, pp. 3-42.

Bowles, Samuel y Herbert Gintis, 1981, La instrucción escolar en la América capitalista: la reforma educativa y las contradicciones de la vida económica, México, Siglo XXI Editores.

Bronk, Kendall Cotton, W. Holmes Finch y Tasneem L. Talib, 2010, "Purpose in Live Among High Ability Adolescents", High Ability Studies, vol. 21, núm. 2, pp. 133145 .

Cerda, Gamal, Rosario Ortega, Carlos Pérez, César Flores y Roberto Melipillán, 2011, “Inteligencia lógica y rendimiento académico en matemáticas: un estudio con estudiantes de Educación Básica y Secundaria de Chile", Anales de Psicología, vol. 27, núm.2, pp. 389-398.

Cole, Michael, 2011, "Reinventando las prácticas educativas del pasado para lograr el éxito pedagógico del futuro", Revista de Estudios Sociales, núm. 40, pp. 23-32.

Cornejo, Rodrigo y Jesús Redondo, 2007, “Variables y factores asociados al aprendizaje escolar: una discusión desde la investigación actual", Estudios Pedagógicos, vol. 33, núm. 2, pp. 155-175.

Davaillon, Alice y Emmanuelle Nauze-Fichet, 2004, “Les trajectoires scolaires des enfants pauvres", Education et Formations, vol. 70, pp. 41-63. 
Ericcson, K. Anders, Roy Roring y Kiruthiga Nandagopal, 2007, “Giftedness and Evidence for Reproducible Superior Performance: an Account Based on the Expert Performance Framework", High Ability Studies, vol., 18, núm. 1, pp. 3-56.

Feyfant, Annie, 2011, “Les effets de l'éducation familiale sur la réussite scolaire", Veille et Analyses, núm. 63, pp. 1-14.

Finn, Jeremy y Rock, Donald, 1997, “Academic Success Among Students at Risk for School Failure”, Journal of Applied Psychology, vol. 82, núm. 2, pp. 221-234.

Gagné, Francoys, 2010, “Motivation within the DMGT 2.0 framework”, High Ability Studies, vol. 21, núm. 2, pp. 81-99.

Giroux, Henri, 2004, Teoría y resistencia en educación: una pedagogía para la oposición, Buenos Aires, 6a. ed., Siglo XXI Editores.

Lahire, Bernard, 1995, Tableaux de familles. Heurs le malherurs scolaires en milieux populaires, Paris, Seuil/Gallimard.

Manciaux, Michel, Stefan Vanistendael, Jacques Lecompte y Boris Cyrulnik, 2003, "La resiliencia: estado de la cuestión", en Michel Manciaux (comp.), La resiliencia: resistir y rehacerse, Barcelona, Gedisa, 17-27.

Martin, Andrew y Herbert Marsh, 2006, “Academic Resilience and its Psychological and Educational Correlates: A Construct Validity Approach", Psychology in the Schools, vol. 43, núm. 3, 267-281.

Mercklé, Pierre, 2005, “Une sociologie des 'irrégularités sociales' est-elle possible?", Idées, la Revue des Sciences Économiques et Sociales, núm. 142, pp. 22-29. Disponible en: 〈http:/ / socio.ens-lsh.fr/merckle_textes_2005_irregularites.pdf».

Murillo, Javier (coord.), 2003, La investigación sobre eficacia escolar en Iberoamérica: revisión internacional sobre el estado del arte, Bogotá, Convenio André Bello/Centro de Investigación y Documentación Educativa.

Neber, Heinz y Marlene Schommer-Aikins, 2002, “Self-regulate Science Learning with Highly Gifted Students: the Role of Cognitive, Motivational, Epistemological and Environmental Variables", High Ability Studies, vol. 13 núm. 1, pp. 59-74.

Organisation for Economic Co-operation and Development (OECD), 2011, Against the Odds: Disadvantaged Students who Succeed in School, OECD Publishing. Disponible en: 〈http//dx.doi.org/10.1787/9789264090873-en〉. 
Organisation for Economic Co-operation and Development (OECD), 2010a, "PISA 2009 Results. What Students Know and Can Do: Student Performance", Reading, Mathematics and Science, vol. 1, OECD Publishing.

Organisation for Economic Co-operation and Development (OECD), 2010b, "PISA 2009 Results: Overcoming Social Background: Equity in Learning Opportunities and Outcomes, Reading, Mathematics and Science, vol. 2, OECD Publishing.

Organisation for Economic Co-operation and Development (OECD), 2010c, "PISA 2009 Results: Learning to Learn: Student Engagement, Strategies and Practices", Reading, Mathematics and Science, vol. 3, OECD Publishing.

Organisation for Economic Co-operation and Development (OECD), 2009, PISA Data analysis manual, SPSS, 2a. ed., OECD Publishing.

Raczynski, Dagmar, Daniel Salinas, Loreto de la Fuente, Macarena Hernández y Miguel Lattz, 2010, Hacia una estrategia de validación de la educación pública municipal: imaginarios, valoraciones y demandas de las familias (informe final de investigación), Santiago, Fondo de Investigación y Desarrollo en Educación/Ministerio de Educación.

Schick, Hella y Shane Phillipson, 2009, “Learning Motivation and Performance Excellence in Adolescents with High Intellectual Potential: What Really Matters? en High Ability Studies, vol. 20, núm. 1, pp. 15-37.

Rama, Germán, 1991, Qué aprenden y quiénes aprenden en las escuelas de Uruguay: los contextos sociales e institucionales de éxitos y fracasos, Montevideo, CEPAL.

Rouse, Kimberly A. Gordon, 2001, “Resilient Student's Goals and Motivation, Journal of Adolescence, núm. 24, pp. 461-472.

Tedesco, Juan Carlos, 2010, “Los temas de la agenda sobre gobierno y dirección de los sistemas educativos en América Latina", en Marchesi, A., Tedesco, J.C. y Coll, C., Calidad, equidad y reformas en la enseñanza, España, OEI-/Fundación Santillana, pp. 77-86.

Recibido el 7 de febrero de 2012 Aceptado el 10 de abril de 2013 